\title{
O Qualis Periódicos e sua utilização nas avaliações
}

\section{The Qualis Periódicos and its use in evaluations}

\section{El Qualis Periódicos y su utilización en las evaluaciones}

http://dx.doi.org/10.21713/2358-2332.2016.v13.1128

Nei Yoshihiro Soma, doutor em Matemática Computacional e Aplicada pela University of Sheffield, EUA, e professor Associado do Instituto Tecnológico de Aeronáutica (ITA), São José dos Campos, SP, Brasil. E-mail: soma@ita.br.

Alexandre Donizeti Alves, doutor em Computação Aplicada pelo Instituto Nacional de Pesquisas Espaciais e bolsista de pósdoutorado do Instituto Tecnológico de Aeronáutica (ITA), São José dos Campos, SP, Brasil. E-mail: adalves@ita.br.

Horacio Hideki Yanasse, Doutor em Pesquisa Operacional pelo Massachusetts Institute of Technology, EUA, e professor titular da Universidade Federal de São Paulo (Unifesp), São José dos Campos, SP, Brasil. E-mail: horacio.yanasse@unifesp.br.

\footnotetext{
"Muito ciosa das conquistas feitas de liberdade de pensamento e de crítica, a Universidade não as dispensa para viver. Não terá ela nenhuma 'verdade' a dar, a não ser a única verdade possivel, que é a de buscá-la eternamente" (ANÍSIO TEIXEIRA, 1962).
}

\section{Resumo}

O Qualis Periódicos é um conjunto de listas contendo títulos de periódicos que tiveram publicações de docentes e discentes dos programas que fazem parte do Sistema Nacional de Pós-Graduação (SNPG). A utilização do Qualis tem ocorrido na apreciação, por parte da Coordenação de Aperfeiçoamento de Pessoal de Nivel Superior (Capes), 
de propostas de cursos novos e na avaliação, com atribuição de notas, de cursos em funcionamento reconhecidos pela Capes. Apresentamse aqui algumas reflexões sobre as elaborações das listas Qualis, uma proposta para se tentar montar uma lista única e, finalmente, a utilização do Qualis em outras avaliações que não aquelas feitas pela Capes.

Palavras-chave: Qualis. Avaliação de Programas de Pós-Graduação. Sistema Nacional de Pós-Graduação.

\section{Abstract}

The Qualis Periódicos is a set of lists containing the titles of journals that had articles published in them by graduate program lecturers, researches and students that compose the Sistema Nacional de Pós-Graduação (Brazilian Graduate System). The use of Qualis Periódicos occurs both to evaluate proposals for new courses and to determine a grade for programs evaluated by the Brazilian Federal Agency for Support and Evaluation of Graduate Education (CAPES). Some reflections are suggested here on the elaboration of the Qualis lists, in an attempt to generate a single list, and on the use of Qualis in distinct evaluations that do not belong to CAPES.

Keywords: Qualis. Graduate Program Evaluation. Brazilian Graduate System.

\section{Resumen}

Qualis Periódicos es un conjunto de listas conteniendo títulos de periódicos que tuvieron publicaciones de profesores, investigadores y alumnos de los programas que forman parte del Sistema Nacional de Postgrado (SNPG). Qualis es utilizado cuando se evalúan las propuestas de nuevos cursos y en la evaluación de los mismos, con atribución de notas, por parte de la Coordinación de Perfeccionamiento de Personal de Nivel Superior (CAPES). Aquí se proponen algunas reflexiones sobre la construcción de las listas Qualis, con la intención de estructurar una lista única, y finalmente utilizar el Qualis en otras evaluaciones que no sólo sean de la CAPES.

Palabras clave: Qualis. Evaluación de Programas de Postgrado. Sistema Brasileño de Postgrado. 


\section{INTRODUCÃ̃O}

O Qualis Periódicos é formado por um conjunto de listas contendo todos os títulos de periódicos que publicaram artigos de docentes e discentes de programas de pós-graduação reconhecidos pela Capes. As listas Qualis são atualizadas anualmente e utilizadas na avaliação dos programas de pós-graduação. O Qualis de periódicos tem sido objeto de grande discussão por parte da comunidade acadêmica do país, e observa-se que todos que possuem alguma relação com a pós-graduação brasileira têm um posicionamento sobre essas listas e o seu uso nas avaliações. Há manifestações sobre esse instrumento em veículos como jornais de circulação nacional ou, ainda, em sites de compartilhamento de vídeos. Discussões ocorrem em diversos fóruns do saber como seções específicas de eventos científicos e em artigos de periódicos das mais diversas áreas do conhecimento. Nesta mesma seção de Debates, há artigo muito interessante, o intitulado Dez coisas que você deveria saber sobre o Qualis que apresenta de maneira lúcida um estudo sobre o Qualis Periódicos, destacando pontos essenciais que permitem seu correto entendimento, bem como o contexto histórico da própria Avaliação de Programas de Pós-Graduação na Capes. Entendese que esse mesmo artigo é de leitura imprescindivel para os envolvidos com a pós-graduação.

Apresentam-se aqui algumas reflexões sobre o papel do Qualis de Periódicos e sua utilização na avaliação da atuação conjunta de grupos de docentes e também dos discentes da pós-graduação vinculados a programas recomendados e reconhecidos pela Capes.

O presente texto tem como ponto de partida os dados informados por docentes e discentes dos programas de pós-graduação, as listas Qualis feitas pela área de avaliação e aprovadas pelo Conselho TécnicoCientífico da Educação Superior (CTC-ES) e sua posterior utilização em diversas ações, incluindo as avaliações realizadas pela Capes - e não somente estas últimas.

Para a elaboração das listas Qualis, as áreas de avaliação utilizam diversas bases de indexação, mas, no presente estudo, serão consideradas apenas quatro dessas bases: Web of Science (WoS), Journal 
Citation Reports(JCR) ambas da Thomson Reuters; Scopus, da Elsevier, e SCImago Journal \& Country Rank, da SCIMago Lab e que também utiliza dados da Scopuse do Ulrich's. Propõe-se uma metodologia na tentativa de se obter uma única lista Qualis, que, neste trabalho, é ilustrada fazendo uso somente da base de indexação JCR, o que limita a lista a títulos de periódicos somente desta base.

\section{OS DADOS NO SISTEMA NACIONAL DE PÓS-GRADUAÇÃO (SNPG)}

Os dados sobre os programas que estão no SNPG têm tramitação digital eletrônica, sejam eles relacionados à avaliação de programas, sejam eles para ingresso no sistema (CAPES, 2014b). Os documentos digitais são tornados públicos após a consolidação das informações. O mesmo ocorre com as decisões tomadas nos diversos órgãos colegiados do Ministério da Educação (MEC). Isso faz com que se tenha a transparência pública dos atos na avaliação da Capes. Aqui é preciso destacar o grande avanço obtido na busca por uma maior transparência pública, a partir do ano de 2014, com o lançamento da Plataforma Sucupira (CAPES, 2014a). O nome da plataforma é uma homenagem ao Prof. Newton Lins Buarque Sucupira (BOMENY, 2001). O Prof. Lívio Amaral, à época Diretor de Avaliação (DAV) da Capes, foi o idealizador dessa plataforma, bem como da concepção de sua integração nos diversos serviços de bancos de dados já existentes no órgão.

Um dos quesitos da avaliação dos programas do SNPG se dá por intermédio da análise da produção intelectual docente e discente. A produção intelectual de um programa é composta por diversos produtos, como, por exemplo, publicação de artigo completo em periódico, software registrado, patente licenciada, comunicação em evento científico, livro publicado, capítulo de livro publicado etc. Para, e tão somente para, a avaliação dos artigos completos publicados, há as listas Qualis Periódicos. Cada lista contém um conjunto de todos os títulos de periódicos que publicaram artigos dos docentes e discentes dos programas de pósgraduação de uma área de avaliação reconhecidos pela Capes. Note-se que os programas são agrupados, por afinidade temática, em áreas de avaliação, sendo que atualmente há 48 delas. As áreas, por sua vez, são agrupadas em nove grandes áreas, e estas, em três colégios de ciência. 
Para percepção e primeira aproximação das dificuldades impostas à avaliação dos artigos completos relatados no SNPG, deve-se ter presente a própria dimensão da pós-graduação brasileira. Em 2014, o SNPG era composto por 3.729 programas, com um corpo docente de 84.242 professores (permanentes, colaboradores e visitantes) e corpo discente de 302.034 alunos (dos mestrados profissional e acadêmico e doutorado) (CAPES, 2016c). No Gráfico 1 apresenta-se a evolução do número de artigos publicados por docentes e discentes desses programas que estão indexados na base WoS, cobrindo um período de 20 anos. Observa-se que somente para o ano de 2014 há quase um artigo com indexação na WoS por docente, considerando o fato de que um mesmo docente pode ter atuação em mais de um programa de pós-graduação (CAPES, 2014c). Um ponto que merece ser destacado na produção científica nacional é a forte correlação entre o volume de artigos em bases indexadas do saber e a consolidação do Portal de Periódicos da Capes. É possível constatar o crescimento do número das publicações a partir de 2007, o que também acompanha o crescimento do próprio Portal no que se refere à quantidade de títulos e bases disponibilizadas para a comunidade do país (CAPES, 2016a).

\section{Gráfico 1 - Evolução do número de artigos de autores do Brasil indexados na WoS}

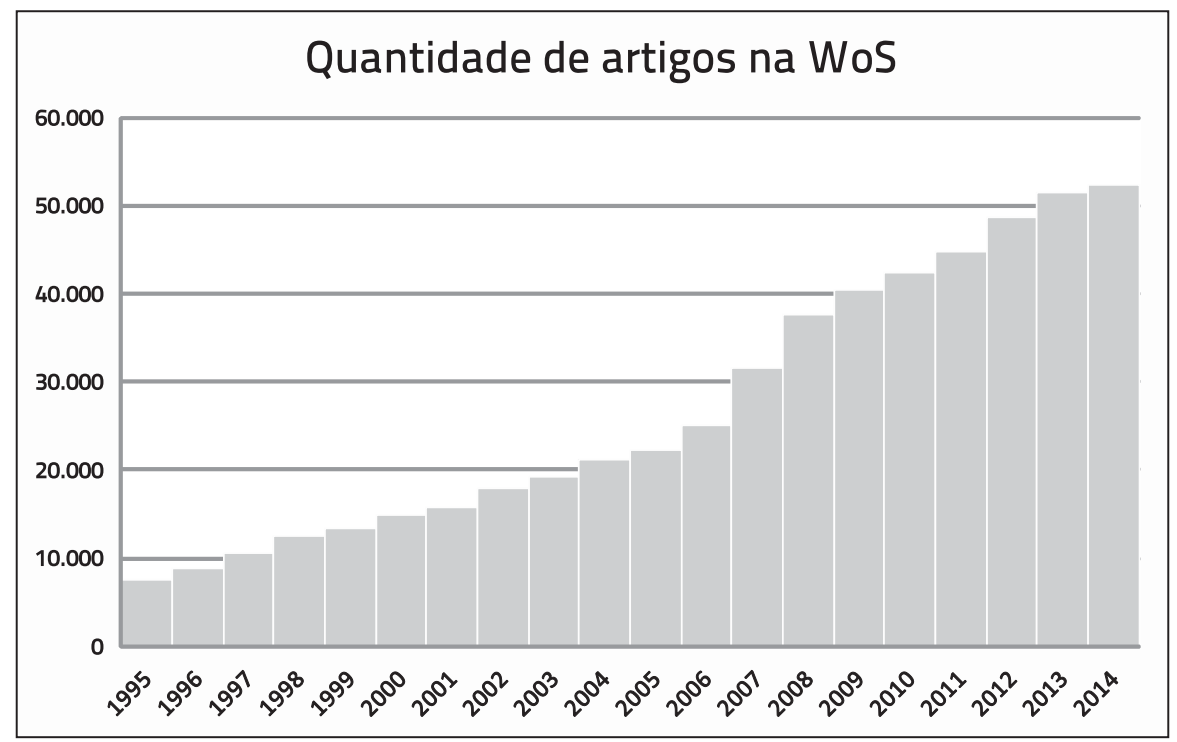

Fonte: WoS 2016 
Os totais por ano das produções apresentadas no Gráfico 1 não são compostos unicamente por artigos em periódicos, eles incluem, embora em menor quantitativo, outros 32 tipos de produções bibliográficas, como, por exemplo, revisão de livros, correções e até mesmo reimpressões de artigos. Se, por um lado, é claro que, para - Qualis Periódicos, somente os artigos em periódicos devam ser considerados; por outro, parece natural se pensar que somente artigos originais devam ser levados em conta nas avaliações dos programas de pós-graduação pela Capes, o que exigiria uma triagem das informações das bases de indexação, seja por parte da DAV, seja por parte dos comitês de área.

\section{A ELABORAÇÃO DAS LISTAS QUALIS}

As listas Qualis são obtidas a partir das informações apresentadas pelos programas do SNPG e da Plataforma Sucupira (PS). Para o caso específico das publicações em periódicos, a Plataforma Lattes, do CNPq, é utilizada para a importação da produção intelectual e para o cruzamento de informações com a PS (CAPES, 2016b). As coordenações de um programa, assim como os pró-reitores de pós-graduação, também atuam para que os dados de seus respectivos programas não contenham equivocos e sejam consistentes. Há processamento, ainda, por parte da DAV para o cadastramento e a homologação de periódico ainda não constante da PS. Em data determinada pela Capes, há um congelamento das informações de todos os programas, e são geradas listas contendo os títulos dos periódicos que tiveram publicações no período de um ano, para cada uma das 48 áreas de avaliação.

Cada área de avaliação deve classificar todos os títulos de periódicos em que artigos de docentes e discentes de programas daquela área foram publicados naquele período. Tal classificação deve se dar em oito estratos, com uma gradação, em ordem crescente de qualificação, a saber: C, B5, B4, B3, B2, B1, A2 e A1. Por exemplo, um título de periódico que está em B1 tem uma qualificação maior do que outro que está no estrato B2, mas tem uma menor qualificação do que outro que está no estrato $\mathrm{A} 2$. $\mathrm{O}$ estrato $\mathrm{C}$ contém títulos que não 
são considerados na avaliação por uma área, como, por exemplo, uma revista de divulgação que não tenha processo de revisão por pares para a aceitação da publicação de seus artigos. À exceção do estrato C, os demais não devem ser vazios. Assim, eventualmente, uma área pode ter seu estrato $C$ vazio, mas necessariamente, os estratos $B 5, B 4, B 3$, $B 2$, B1, $A 2$, e $A 1$ não o podem.

É importante ressaltar que, se um periódico tem uma qualificação Qualis maior do que outro por estar em estrato distinto, não significa que o do estrato mais alto tem maior qualidade técnica ou científica do que o do outro. Igualmente, se dois periódicos estiverem em um mesmo estrato, não significa que os artigos destes dois possuem a mesma qualidade técnica ou científica. A estratificação dos periódicos por uma dada área na Capes nesses oito estratos só, e somente só, é feita para que seja possivel realizar a avaliação comparativa dos programas de pós-graduação constantes do SNPG, mais especificamente, para que seja possível avaliar a produção intelectual comparativa entre programas de uma mesma área na Capes, segundo regras estabelecidas por este órgão. Ressalta-se que a produção intelectual é apenas um entre cinco quesitos da avaliação (CAPES, 2014b).

Além de ser preciso atribuir a um título de periódico - que teve publicação no período na área - um estrato, há somente as seguintes regras a serem obedecidas:

- A quantidade de títulos no estrato A1 não deve ser maior do que a do estrato $A 2$;

- A quantidade de títulos nos estratos A1 e A2 não deve ultrapassar, no conjunto, a 25\% do total de títulos de periódicos da área naquele ano;

- A quantidade de títulos nos estratos A1, A2 e B1 não deve ultrapassar, no conjunto, a 50\% do total de títulos de periódicos da área naquele ano;

- Nenhum estrato deve estar vazio, com a exceção do estrato C.

Os títulos no estrato C não são considerados no total de títulos, pois, conforme mencionado anteriormente, o estrato C contém títulos 
que não são considerados na avaliação pela área. Deve ser lembrado uma vez mais, dada a quantidade de críticas recebidas pela inserção de títulos nos estratos, que o contexto é tão somente o da avaliação dos programas na Capes. Não se deve usar as listas Qualis para avaliar a qualidade de periódicos ou o desempenho individual de pessoas. Este último ponto será retomado mais adiante.

Cada área de avaliação elabora critérios próprios para explicitar como se dá a alocação dos títulos nos estratos, em adição às regras gerais relacionadas aos percentuais de títulos nos estratos que devem ser seguidas. A lista Qualis de um ano (CAPES, 2016d) e a descrição da metodologia daquela alocação, Arquivo Qualis 2016 nas páginas das áreas (CAPES, 2014b), são objeto de deliberação por parte do CTC-ES para que possam ser tornados públicos. Como exemplos de alocações em estratos de títulos de periódicos publicados pelos programas no biênio 2013-2014, citamos: na área de Farmácia, em A1, estão os periódicos que possuem Fator de Impacto (FI), na base JCR, maior ou igual a 4; para a de Ciências Biológicas II, o FI deve ser maior ou igual a 4,634; para as Engenharias III, em B2 e B3 estão aqueles que estão na base Scopus, mas não na base JCR, e a classificação dos títulos é por ordem decrescente do indicador SJR (SCImago Journa/ Rank); para a Odontologia, em B4 estão os títulos que estejam indexados em ao menos uma das seguintes bases: Lilacs, Latindex ou Ebsco.

As áreas de avaliação utilizam diversas bases de indexação, sendo que a própria consulta ao ISSN dos periódicos pode ser realizada por meio de diversas delas. Para a consulta aos ISSNs, neste trabalho, utilizou-se a base Ulrich's, que apresenta grande quantidade de informações, como por exemplo, as bases de indexação e a de resumos.

Na Tabela 1 tem-se os percentuais de títulos de periódicos das áreas, das grandes áreas e dos colégios de ciências que não constam nas bases JCR, Scopuse SC/mago. Também, há os percentuais de títulos que não são considerados na avaliação pelas áreas. 
Tabela 1 - Percentuais de títulos de periódicos sem indexação ou no estrato $C$

\begin{tabular}{|c|c|c|c|c|c|c|}
\hline Ärea 2013/2014 & s.Ind & C & Grande Ārea & s.Ind/C & $\begin{array}{c}\text { Colégio de } \\
\text { Ciências }\end{array}$ & s.ind/C \\
\hline Ciência de Alimentos & $8 \%$ & $21 \%$ & \multirow{4}{*}{ Agrárias } & \multirow{4}{*}{$\begin{array}{l}20 \% \\
(14 \%)\end{array}$} & \multirow{17}{*}{ Vida } & \multirow{17}{*}{$\begin{array}{l}18 \% \\
(9 \%)\end{array}$} \\
\hline Ciências Agrárias I & $30 \%$ & $8 \%$ & & & & \\
\hline Medicina Veterinária & $27 \%$ & $0 \%$ & & & & \\
\hline $\begin{array}{l}\text { Zootecnia e Recursos } \\
\text { Pesqueiros }\end{array}$ & $14 \%$ & $25 \%$ & & & & \\
\hline Biodiversidade & $4 \%$ & $24 \%$ & \multirow{4}{*}{ Biológicas } & \multirow{4}{*}{$\begin{array}{c}5 \% \\
(15 \%)\end{array}$} & & \\
\hline Ciências Biológicas I & $13 \%$ & $1 \%$ & & & & \\
\hline Ciências Biológicas II & $2 \%$ & $13 \%$ & & & & \\
\hline Ciências Biológicas III & $2 \%$ & $22 \%$ & & & & \\
\hline Educação Física & $33 \%$ & $0 \%$ & \multirow{9}{*}{ Saúde } & \multirow{9}{*}{$\begin{array}{l}22 \% \\
(5 \%)\end{array}$} & & \\
\hline Enfermagem & $47 \%$ & $2 \%$ & & & & \\
\hline Farmácia & $7 \%$ & $11 \%$ & & & & \\
\hline Medicina I & $13 \%$ & $4 \%$ & & & & \\
\hline Medicina II & $15 \%$ & $4 \%$ & & & & \\
\hline Medicina III & $9 \%$ & $8 \%$ & & & & \\
\hline Nutrição & $17 \%$ & $4 \%$ & & & & \\
\hline Odontologia & $30 \%$ & $2 \%$ & & & & \\
\hline Saúde Coletiva & $30 \%$ & $6 \%$ & & & & \\
\hline Astronomia e Física & $2 \%$ & $18 \%$ & \multirow{5}{*}{$\begin{array}{l}\text { Ciências } \\
\text { Exatas e } \\
\text { da Terra }\end{array}$} & \multirow{5}{*}{$\begin{array}{c}10 \% \\
(13 \%)\end{array}$} & \multirow{14}{*}{$\begin{array}{c}\text { Exatas } \\
\text { Tecnológicas } \\
\text { e } \\
\text { Multidisci- } \\
\text { plinar }\end{array}$} & \multirow{14}{*}{$\begin{array}{l}24 \% \\
(9 \%)\end{array}$} \\
\hline Ciências da Computação & $6 \%$ & $22 \%$ & & & & \\
\hline Geociências & $23 \%$ & $6 \%$ & & & & \\
\hline Matemática, Prob. e Est. & $16 \%$ & $1 \%$ & & & & \\
\hline Química & $4 \%$ & $18 \%$ & & & & \\
\hline Biotecnologia & $9 \%$ & $12 \%$ & \multirow{5}{*}{$\begin{array}{l}\text { Multidisci- } \\
\text { plinar }\end{array}$} & \multirow{5}{*}{$\begin{array}{l}34 \% \\
(8 \%)\end{array}$} & & \\
\hline Ciências Ambientais & $41 \%$ & $5 \%$ & & & & \\
\hline Ensino & $62 \%$ & $4 \%$ & & & & \\
\hline Interdisciplinar & $48 \%$ & $5 \%$ & & & & \\
\hline Matérias & $10 \%$ & $17 \%$ & & & & \\
\hline Engenharias I & $31 \%$ & $13 \%$ & \multirow{4}{*}{ Engenharias } & \multirow{4}{*}{$\begin{array}{l}28 \% \\
(5 \%)\end{array}$} & & \\
\hline Engenharias II & $27 \%$ & $0 \%$ & & & & \\
\hline Engenharias III & $32 \%$ & $4 \%$ & & & & \\
\hline Engenharias IV & $22 \%$ & $3 \%$ & & & & \\
\hline
\end{tabular}




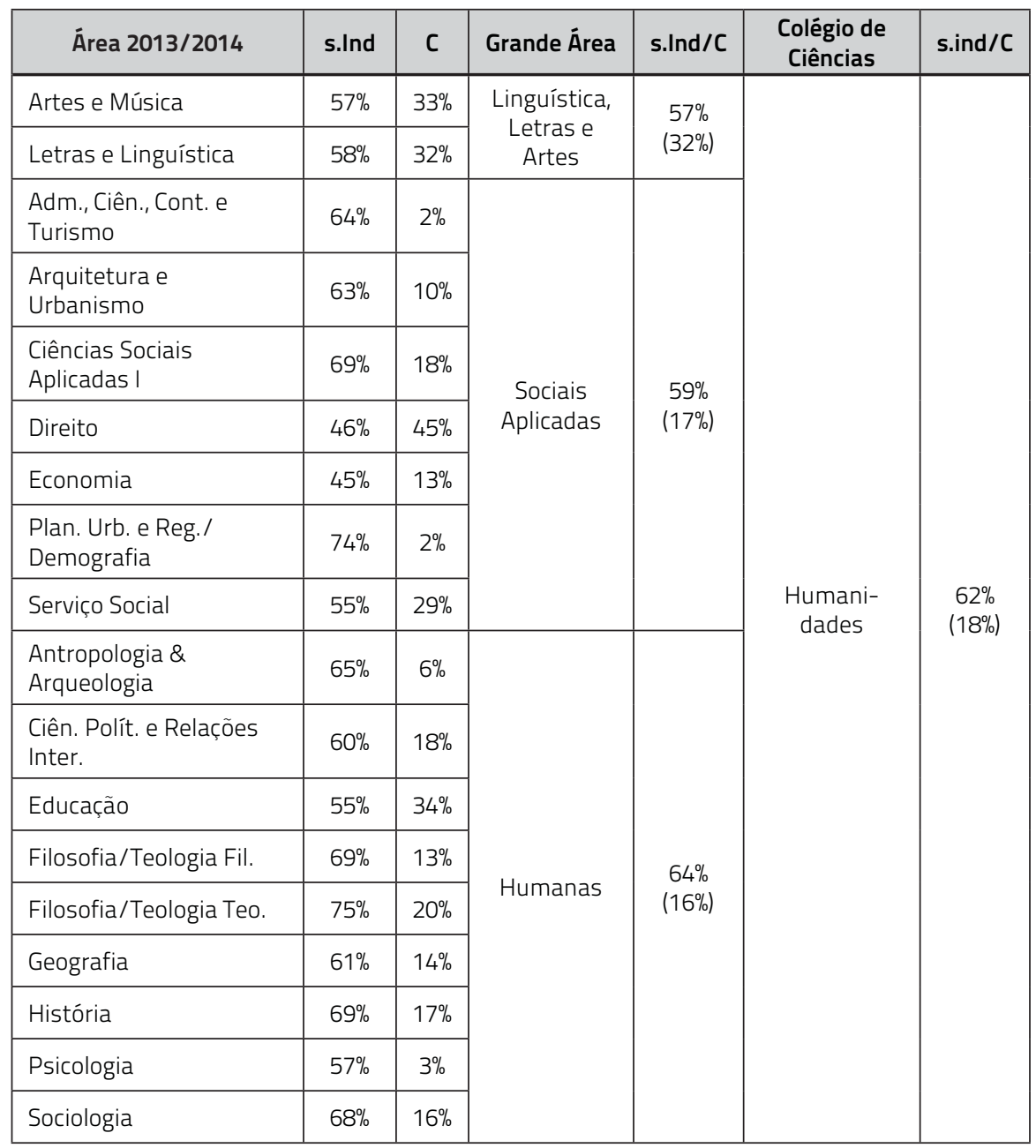

Fonte: elaboração própria

Na Tabela 1, a coluna s.Ind. (sem indicação) dá o percentual de títulos que não estão indexados em nenhuma das três bases, JCR, Scopus e SClmago. Deve ser observado o fato de que as bases diferem em seu conteúdo (ALVES et al., 2014), por exemplo, para o ano de 2014, o periódico Academia (Caracas) tem os mesmos indicadores no SCImago e JCR, mas, no Scopus, seu ISSN consta como inativo. Fazendo uma consulta ao Ulrich's, também não há a indexação do periódico em 2014. Nas colunas $S$.ind/C, apresenta-se a média expressa em percentuais de títulos sem indexação e em C, esta última entre parênteses, para as grandes áreas e os colégios de ciência.

Observa-se na Tabela 1 que as áreas de Medicina Veterinária, Educação Física e Engenharias II não tiveram periódico algum no 
estrato C e, aproximadamente, 30\% dos títulos dos periódicos das três áreas não estão indexadas nas bases JCR, Scopuse SCImago. Por outro lado, é possivel perceber que há 11 áreas em que o percentual de não indexação é menor do que o do estrato C, com destaque para as áreas de Ciências Biológicas III e de Astronomia e Física, que têm em torno de 20\% dos títulos no estrato C e 2\% de títulos sem indexação. Também, é possivel notar aqui que a utilização de tão somente as bases JCR, Scopus e SClmago não é adequada para o Colégio de Humanidades.

\section{QUALIS ÚNICO}

Caso as listas Qualis de todas as áreas, para os anos de 2013 e 2014, fossem consolidadas em uma única listagem, se contabilizarmos somente os ISSNs distintos, o resultado seria quase 17.000 títulos (20\% do total), número maior do que a quantidade de títulos constantes do JCR edição Science ou edição Social.

Um dos grandes desafios para se montar uma única lista Qualis para todas as áreas ou um grande grupo de áreas reside na disparidade de classificação de periódicos nos diferentes estratos observados nas diferentes áreas de avaliação. Existem muitos títulos que aparecem em listas Qualis de diferentes áreas. Por exemplo, para os anos de 2013 e 2014, os periódicos que aparecem em mais listas Qualis de diferentes áreas são: Arquivo Brasileiro de Medicina Veterinária e Zootecnia, Journal of the Brazilian Chemical Society, Molecules, Brazilian Archives of Biology and Technology, Genetics and Molecular Research, Holos, Revista GEINTEC: Gestão, Inovação e Tecnologias, Anais da Academia Brasileira de Ciências, BioMed Research International, The Scientific World Journal, Scientia Plena, Revista Sodebras, Cadernos de Saúde Pública, Ciência e Saúde Coletiva, e PLOS One. Esses títulos que aparecem em listas Qualis de diversas áreas diferentes muitas vezes são classificados em estratos distintos. Como exemplo, é possivel citar o periódico PLOS One, que aparece nos estratos C, B2, B1, A2 e A1. A Revista Brasileira de Estudos Pedagógicos, que publicou o discurso do Prof. Anísio Teixeira de 1935, citado no início deste artigo, aparece nos estratos C, B5, B3, B2, B1 e A2. Há muitos outros exemplos que mostram que as variações na 
classificação nos estratos são grandes entre as áreas. O artigo da Profa. Rita Barradas Barata também destaca o problema da disparidade de classificações entre periódicos como uma dificuldade para a elaboração de uma lista Qualis única.

Existem, no entanto, iniciativas já em uso por algumas áreas de avaliação que visam mitigar o problema da disparidade de classificação de periódicos utilizados por docentes e discentes que atuam em subáreas do conhecimento dentro de uma mesma área de avaliação. Tais iniciativas poderiam ser eventualmente estendidas para a classificação de periódicos de uma grande área ou grupos de áreas de avaliação.

Apresenta-se, a seguir, parte da metodologia da área de Engenharias III, que, ocasionalmente, pode se mostrar útil para outras áreas de avaliação. Primeiro, o comitê Qualis da área reconheceu que havia um problema: como comparar o perfil de publicações de suas diversas subáreas do conhecimento? Por exemplo, a Engenharia Naval e Oceânica (cujos programas estão na área) é uma subárea que contém somente 14 títulos de periódicos no JCR, enquanto que a Engenharia Mecânica, outra subárea da Engenharias III, tem 130 títulos de periódicos na mesma base, de acordo com dados do ano de 2014. Uma utilização direta do fator de impacto faria com que os títulos dos periódicos da Engenharia Naval e Oceânica ficassem, no máximo, no estrato B1. Esse tipo de comparação já foi considerado errado, inclusive pelo próprio criador do JCR (GARFIELD, 1979). A saída encontrada foi a de relativizar os diversos fatores de impacto entre subáreas pela mediana das subáreas. Essa proposta foi sugerida pelo Prof. Adiel Teixeira de Almeida, à época coordenador adjunto da área. O próprio JCR faz o cálculo das medianas das subáreas (category) que o compõem. A divisão do fator de impacto de um periódico pela mediana de sua subárea, chamado de Fator de Impacto Relativizado (FIR), faz com que a ordem relativa entre os periódicos de uma mesma subárea seja inalterada, mas com o benefício de propiciar uma maneira de se comparar subáreas diferentes, utilizando, por exemplo, os quartis observados. Para o exemplo em questão, a subárea (category) Engineering, Marine tem mediana 0,483, enquanto que a mediana para (category) Engineering, Mechanicalé de 0,956. Se for feita essa relativização, a subárea de Engenharia Naval e Oceânica 
terá quatro periódicos no primeiro quartil, e a Engenharia Mecânica 36, sendo que, neste caso e de maneira proporcional, essas quantidades de periódicos nas respectivas subáreas correspondem a $28,5 \%$ e $24,6 \%$ do total.

A Engenharias III considera também a "meia-vida" do periódico em sua metodologia para elaboração de sua lista Qualis, mas a sua utilização nas demais áreas de avaliação precisaria ser mais bem estudada.

No tabela a seguir, tem-se os percentuais de títulos de periódicos nos estratos mais altos do Qualis, B2, B1, A2 e A1, de cada área e que possuem indexação no JCR.

Tabela 2 - Percentuais de utilização da base JCR nos estratos de B2 a A1

\begin{tabular}{|c|c|c|c|c|c|c|c|c|}
\hline Área 2013/2014 & B2_A1 & B1_A1 & Grande Área & B2_A1 & B1_A1 & $\begin{array}{c}\text { Colégio de } \\
\text { Ciências }\end{array}$ & B2_A1 & B1_A1 \\
\hline Ciência de Alimentos & $93 \%$ & $94 \%$ & \multirow{4}{*}{ Agrárias } & \multirow{4}{*}{$85,0 \%$} & \multirow{4}{*}{$89,7 \%$} & \multirow{17}{*}{ Vida } & \multirow{17}{*}{$84 \%$} & \multirow{17}{*}{$89 \%$} \\
\hline Ciências Agrárias I & $76 \%$ & $88 \%$ & & & & & & \\
\hline Medicina Veterinária & $88 \%$ & $89 \%$ & & & & & & \\
\hline $\begin{array}{l}\text { Zootecnia e Recursos } \\
\text { Pesqueiros }\end{array}$ & $84 \%$ & $87 \%$ & & & & & & \\
\hline Biodiversidade & $86 \%$ & $88 \%$ & \multirow{4}{*}{ Biológicas } & \multirow{4}{*}{$89,9 \%$} & \multirow{4}{*}{$91,1 \%$} & & & \\
\hline Ciências Biológicas I & $93 \%$ & $93 \%$ & & & & & & \\
\hline Ciências Biológicas II & $88 \%$ & $90 \%$ & & & & & & \\
\hline Ciências Biológicas III & $92 \%$ & $92 \%$ & & & & & & \\
\hline Educação Física & $70 \%$ & $80 \%$ & \multirow{9}{*}{ Saúde } & \multirow{9}{*}{$80,5 \%$} & \multirow{9}{*}{$86,9 \%$} & & & \\
\hline Enfermagem & $50 \%$ & $70 \%$ & & & & & & \\
\hline Farmácia & $90 \%$ & $93 \%$ & & & & & & \\
\hline Medicina I & $85 \%$ & $87 \%$ & & & & & & \\
\hline Medicina II & $86 \%$ & $90 \%$ & & & & & & \\
\hline Medicina III & $98 \%$ & $98 \%$ & & & & & & \\
\hline Nutrição & $91 \%$ & $93 \%$ & & & & & & \\
\hline Odontologia & $75 \%$ & $83 \%$ & & & & & & \\
\hline Saúde Coletiva & $79 \%$ & $87 \%$ & & & & & & \\
\hline
\end{tabular}




\begin{tabular}{|c|c|c|c|c|c|c|c|c|}
\hline Área 2013/2014 & B2_A1 & B1_A1 & Grande Área & B2_A1 & B1_A1 & $\begin{array}{l}\text { Colégio de } \\
\text { Ciências }\end{array}$ & B2_A1 & B1_A1 \\
\hline Astronomia e Física & $92 \%$ & $90 \%$ & \multirow{5}{*}{$\begin{array}{l}\text { Ciências } \\
\text { Exatas } \\
\text { e da Terra }\end{array}$} & \multirow{5}{*}{$87,5 \%$} & \multirow{5}{*}{$89,4 \%$} & \multirow{14}{*}{$\begin{array}{l}\text { Exatas, } \\
\text { Tecnológicas } \\
\text { e Multi- } \\
\text { disciplinar }\end{array}$} & \multirow{14}{*}{$69 \%$} & \multirow{14}{*}{$74 \%$} \\
\hline Ciências da Computação & $85 \%$ & $86 \%$ & & & & & & \\
\hline Geociências & $81 \%$ & $88 \%$ & & & & & & \\
\hline Matemática, Prob. e Est. & $88 \%$ & $89 \%$ & & & & & & \\
\hline Química & $92 \%$ & $94 \%$ & & & & & & \\
\hline Biotecnologia & $89 \%$ & $91 \%$ & \multirow{5}{*}{$\begin{array}{l}\text { Multidisci- } \\
\text { plinar }\end{array}$} & \multirow{5}{*}{$65,3 \%$} & \multirow{5}{*}{$69,9 \%$} & & & \\
\hline Ciências Ambientais & $66 \%$ & $84 \%$ & & & & & & \\
\hline Ensino & $29 \%$ & $13 \%$ & & & & & & \\
\hline Interdisciplinar & $54 \%$ & $68 \%$ & & & & & & \\
\hline Materiais & $88 \%$ & $93 \%$ & & & & & & \\
\hline Engenharias I & $78 \%$ & $93 \%$ & \multirow{4}{*}{ Engenharias } & \multirow{4}{*}{$84,0 \%$} & \multirow{4}{*}{$92,3 \%$} & & & \\
\hline Engenharias II & $87 \%$ & $92 \%$ & & & & & & \\
\hline Engenharias III & $83 \%$ & $94 \%$ & & & & & & \\
\hline Engenharias IV & $88 \%$ & $90 \%$ & & & & & & \\
\hline Artes e Música & $5 \%$ & $6 \%$ & \multirow{2}{*}{$\begin{array}{l}\text { Linguística, } \\
\text { Letras e Artes }\end{array}$} & \multirow{2}{*}{$5,7 \%$} & \multirow{2}{*}{$6,4 \%$} & \multirow{18}{*}{$\begin{array}{l}\text { Humani- } \\
\text { dades }\end{array}$} & \multirow{18}{*}{$21 \%$} & \multirow{18}{*}{$27 \%$} \\
\hline Letras e Linguística & $7 \%$ & $7 \%$ & & & & & & \\
\hline $\begin{array}{l}\text { Adm., Ciên., Cont. e } \\
\text { Turismo }\end{array}$ & $36 \%$ & $50 \%$ & \multirow{7}{*}{$\begin{array}{c}\text { Sociais } \\
\text { Aplicadas }\end{array}$} & \multirow{7}{*}{$27,8 \%$} & \multirow{7}{*}{$34,9 \%$} & & & \\
\hline Arquitetura e Urbanismo & $37 \%$ & $45 \%$ & & & & & & \\
\hline Ciências Sociais Aplicadas I & $9 \%$ & $12 \%$ & & & & & & \\
\hline Direito & $10 \%$ & $13 \%$ & & & & & & \\
\hline Economia & $61 \%$ & $73 \%$ & & & & & & \\
\hline $\begin{array}{l}\text { Plan. Urb. e Reg./ } \\
\text { Demografia }\end{array}$ & $22 \%$ & $29 \%$ & & & & & & \\
\hline Serviço Social & $20 \%$ & $22 \%$ & & & & & & \\
\hline Antropologia \& Arqueologia & $28 \%$ & $31 \%$ & \multirow{9}{*}{ Humanas } & \multirow{9}{*}{$19,2 \%$} & \multirow{9}{*}{$24,7 \%$} & & & \\
\hline $\begin{array}{l}\text { Ciên. Polít. e Relações } \\
\text { Inter. }\end{array}$ & $24 \%$ & $40 \%$ & & & & & & \\
\hline Educação & $8 \%$ & $10 \%$ & & & & & & \\
\hline Filosofia/Teologia Fil. & $10 \%$ & $10 \%$ & & & & & & \\
\hline Filosofia/Teologia Teo. & $1 \%$ & $1 \%$ & & & & & & \\
\hline Geografia & $32 \%$ & $40 \%$ & & & & & & \\
\hline História & $9 \%$ & $11 \%$ & & & & & & \\
\hline Psicologia & $43 \%$ & $57 \%$ & & & & & & \\
\hline Sociologia & $16 \%$ & $23 \%$ & & & & & & \\
\hline
\end{tabular}

Fonte: elaboração própria. 
As colunas B2_A1 e B1_A1 indicam os percentuais de títulos indexados no JCR e que estão nos estratos de B2 a $A 1$ e de $B 1$ a $A 1$, respectivamente. $O$ elevado percentual observado indica que o JCR pode ser usado para discriminar as produções dos colégios de Ciências da Vida e Exatas, Tecnológicas e Multidisciplinares, este último, com a exceção da área de Ensino. A utilização somente do JCR é uma opção claramente inadequada para as áreas de Ciências Sociais Aplicadas I, Direito, Educação, Ensino e Filosofia/Teologia, e para o Colégio de Ciências das Humanidades.

A utilização do FIR pode vir a ser uma opção viável para a montagem de uma lista Qualis única para os colégios de Ciências da Vida e Exatas, Tecnológicas e Multidisciplinares. Há, no entanto, outro ponto que também deveria ser levado em conta na montagem das listas Qualis: a quantidade de artigos publicados nos periódicos em cada uma das áreas. Para ficar com o exemplo já apresentado, o PLOS One, que está em cinco estratos diferentes, se a distribuição da quantidade de publicações nas áreas fosse levada em conta, talvez fosse possivel ter um maior consenso sobre qual seria o estrato que melhor o qualificasse para a utilização na avaliação da Capes.

\section{CONSIDERAÇÕES FINAIS}

A despeito dos alertas frequentemente feitos pela Capes, observa-se que as listas Qualis têm sido sistematicamente utilizadas em outras avaliações, feitas em algumas instituições de ensino e de pesquisa e em algumas agências de fomento para avaliar a qualidade científica da produção de indivíduos, e estão incluídas em critérios para promoção, além de serem utilizadas em comparações da produção científica de qualidade de indivíduos ou de grupos etc. O seu uso, sem a devida ciência de como foram montadas e, consequentemente, de suas limitações, pode ser inadequado, principalmente quando o foco da avaliação está na qualidade de uma produção. Como já ressaltado, a qualificação dos periódicos nos diversos estratos Qualis da Capes não tem uma associação direta com a qualidade técnica ou científica dos periódicos, haja vista as diferentes metodologias utilizadas para a 
elaboração das listas Qualis. Espera-se que os estudos apresentados, como o presente artigo e o da Profa. Rita Barata constante desta edição da $R B P G$, representem uma contribuição ao processo dinâmico do entendimento, da montagem e da utilização do Qualis Periódicos na CAPES.

\section{Referências}

ALVES, A. D.; YANASSE, H. H.; SOMA, N. Y. Benford's law and articles of scientific journals: Comparison of JCR and Scopus data. Scientometrics, v. 98, n. 1, p. 173-184, 2014.

BARATA, R. B. Dez coisas que você deveria saber sobre o Qualis. RBPG, Brasília, v.37, n.1, jan./abr. 2016. no prelo.

BOMENY, H. Newton Sucupira e os rumos da educação superior. Brasília: Paralelo 15, Coordenação de Pessoal de Nivel Superior, Brasília, 2001.

CAPES - Coordenação de Pessoal de Nivel Superior. Capeslança Plataforma Sucupira paragestãoda pós-graduação. 2014a. Disponivel em: <http:// www. capes.gov.br/36-noticias/6810-capes-lanca-plataforma-sucupirapara-gestao-da-pos-graduacao>. Acesso em: maio 2016.

Documento de Área, Relatório de Avaliação, Arquivo Qualis, e Critérios de APCN 2016, nas áreas de Avaliação. 2014b. Disponivel em: <http://capes.gov.br/avaliacao/sobre-as-areas-de-avaliacao/paginasdas-areas>. Acesso em: maio 2016.

Portaria CAPES 174, 2014. 2014c. Disponivel em: <http:// www.capes.gov.br/images/documentos/Portaria_174_2014_ CategoriasDocentesPPG.pdf>. Acesso em: maio 2016.

Histórico do Portal de Periódicos da CAPES, 2016. 2016a. Disponível em: <http://www. periodicos.capes.gov.br/index.php?option=com_ pcontent\&view=pcontent\&alias=historico\&ltemid=100>. Acesso em: maio 2016. 
Manuais da Plataforma Sucupira: Proposta de Cursos Novos e Coleta. 2016b. Disponivel em: <https://sucupira.capes.gov.br/sucupira/ public/lancamento/manual.jsf $>$. Acesso em: maio 2016.

GEOCAPES, 2016. 2016c. Disponivel em: <http://geocapes.capes. gov.br/geocapes2/>. Acesso em: maio 2016.

Qualis Periódicos, CAPES. 2016d. Disponivel em: <https:// sucupira.capes.gov.br/sucupira/public/consultas/coleta/ veiculoPublicacaoQualis/listaConsultaGeralPeriodicos.jsf>. Acesso em: maio 2016.

GARFIELD, E. Is citation analysis a legitimate evaluation tool? Scientometrics, v. 1, n. 4, p. 359-375, 1979.

SCIMAGO JOURNAL \& COUNTRY RANK - SJR. SCImago Journal \& Country Rank. 2016. Disponivel em:<http://www.scimagojr.com/>. Acesso em: maio 2016.

SCOPUS (Elsevier). Scopus. 2016. Disponivel em: <https:/ / www.scopus. com/>. Acesso em: maio 2016.

TEIXEIRA, A. Notas para a história da educação. Revista Brasileira de Estudos Pedagógicos, Rio de Janeiro, v. 37, n. 85, jan./mar. 1962. p.181188. Disponivel em: <http://www. bvanisioteixeira.ufba.br/artigos/ notas2.html>. Acesso em: maio 2016.

ULRICH'S. UlrichsWeb Global Serials Directory. 2016. Disponivel em: <http:/ /www.ulrichsweb.com>. Acesso em: maio 2016.

WOS. Web of Science. 2016. Disponivel em: <https:// webofknowledge. com/>. Acesso em: maio 2016.

Recebido em 28/06/2016 Aprovado em 02/08/2016 